NSF-ITP-99-117

hep-th/9910098

\title{
Baldness/delocalization in intersecting brane systems
}

\author{
Amanda W. Peet \\ Institute for Theoretical Physics, University of California, Santa Barbara, CA \\ 93106-4030, U.S.A.
}

\begin{abstract}
Marginally bound systems of two types of branes are considered, such as the prototypical case of $\mathrm{D} p+4$ branes and $\mathrm{D} p$ branes. As the transverse separation between the two types of branes goes to zero, different behaviour occurs in the supergravity solutions depending on $p$; no-hair theorems result for $p \leq 1$ only. Within the framework of the AdS/CFT correspondence, these supergravity no-hair results are understood as dual manifestations of the Coleman-Mermin-Wagner theorem. Furthermore, the rates of delocalization for $p \leq 1$ are matched in a scaling analysis.

Contribution to the proceedings of "Strings '99"; based on [1] written with D. Marolf.
\end{abstract}

\section{Introduction}

No-hair theorems appear prominently in the study of black holes. They are, however, not general; assumptions about the field content of the action are required.

In the supergravity approximation to string theory, there are black $p$-brane solutions available as well as black holes. Certain systems involving two types of BPS branes are marginally bound, stable at any transverse separation. Supergravity solutions which are fully localized are known for some of these systems, albeit rarely in terms of elementary functions. In this article we will use the method of Surya and Marolf [2] to exhibit the behaviour of the solutions as the separation goes to zero, and to derive no-hair theorems.

The AdS/CFT correspondence [3, 24] is a duality between gauge theories on $p$ branes and the near-horizon limit of the corresponding supergravity solutions. We discuss this limit of the supergravity solutions for the intersecting brane systems of interest, and we find the dual phenomenon to the no-hair theorems to be the ColemanMermin-Wagner theorem [4 5], viz. the lack of superselection sectors in low-dimensional quantum field theories. We also show that the delocalization rates obtained from a scaling analysis in the field theory are captured by the supergravity; in particular, the strong sensitivity to the dimension $p$. We end with remarks about future directions. 


\section{Baldness, a.k.a. supergravity no-hair theorems}

One context in which a black hole no-hair theorem in General Relativity may be seen is a process in which a small extremal black hole of charge $q$ is brought up to the horizon of a large extremal black hole of charge $Q \gg q$. Since the geometry for the two extremal black holes is static for any finite separation $\boldsymbol{\Delta}$, this system can be easily studied at any $\boldsymbol{\Delta}$. As the separation $\boldsymbol{\Delta} \rightarrow 0$, the configuration becomes spherically symmetric. The theory does not allow the small charge to end up localized on the horizon of the large black hole.

More generally, finding localized solutions in supergravity is difficult. As a very simple example, consider taking the $\mathrm{D} p$-brane supergravity solution and performing T-duality along a worldvolume direction, say $x_{\mathrm{p}}$. Use of the Büscher rules results in a solution that looks like a $\mathrm{D} p$-1-brane, except that it possesses only a transverse $\mathrm{SO}(8-p)$ symmetry instead of $\mathrm{SO}(9-p)$. In other words, the harmonic function falls off like $r^{p-7}$ instead of $r^{p-8}$. This solution corresponds to the smeared $\mathrm{D} p-1$-brane. Of course, we know what the unsmeared D $p$-1-brane solution looks like in this case, but more generally the unsmeared version of a smeared solution cannot be successfully guessed. The point of view which we advocate in this article is that sometimes unsmeared supergravity solutions do not in fact exist, for reasons that become clear in the context of the generalized AdS/CFT correspondence.

In the following analysis we are interested in BPS systems comprising two clumps of branes which are marginally bound to each other. The prototype of this system is the D0 $\| \mathrm{D} 4(0)$, and under dualities this is connected to $\mathrm{D} p \| \mathrm{D} p+4, \mathrm{D} m \perp \mathrm{D} n(p), m+n=p+4$ e.g. $\mathrm{D} 2 \perp \mathrm{D} 2(0), \mathrm{F} 1 \perp \mathrm{D} p(0), \mathrm{D} p \perp \mathrm{NS} 5(p-1)$, and so on.

Supergravity solutions for such systems have been studied by many groups; a partial list is [6]-17], 18], see [1] for other references. For purposes of orientation we briefly review the features relevant for our discussion here. Maintaining some generality for the moment, we let there be two types of branes, $\mathrm{D} A$ and $\mathrm{D} B$, with common directions $t, \boldsymbol{z}_{\mathrm{I}}$, relatively transverse worldvolume directions $\boldsymbol{z}_{\mathrm{a}}, \boldsymbol{z}_{\mathrm{b}}$ respectively, and overall transverse coordinates $\boldsymbol{x}_{\perp}$. We use the "harmonic function rule" [10] ansatz; suppressing the R-R gauge potentials this is

$$
\begin{aligned}
\mathrm{d} s^{2}= & H_{\mathrm{A}}^{-1 / 2} H_{\mathrm{B}}^{-1 / 2}\left(-\mathrm{d} t^{2}+\mathrm{d} \boldsymbol{z}_{\mathrm{I}}^{2}\right)+H_{\mathrm{A}}^{1 / 2} H_{\mathrm{B}}^{-1 / 2}\left(\mathrm{~d} \boldsymbol{z}_{\mathrm{b}}\right)^{2} \\
& +H_{\mathrm{B}}^{1 / 2} H_{\mathrm{A}}^{-1 / 2}\left(\mathrm{~d} \boldsymbol{z}_{\mathrm{a}}\right)^{2}+H_{\mathrm{A}}^{1 / 2} H_{\mathrm{B}}^{1 / 2}\left(\mathrm{~d} \boldsymbol{x}_{\perp}^{2}\right) \\
e^{\Phi}= & g_{s} H_{\mathrm{A}}^{(3-A) / 4} H_{\mathrm{B}}^{(3-B) / 4}
\end{aligned}
$$

Allowing the branes to have harmonic functions that depend on all coordinates transverse to them, i.e. $H_{\mathrm{A}}=H_{\mathrm{A}}\left(\boldsymbol{x}_{\perp}, \boldsymbol{z}_{\mathrm{b}}\right), H_{\mathrm{B}}=H_{\mathrm{B}}\left(\boldsymbol{x}_{\perp}, \boldsymbol{z}_{\mathrm{a}}\right)$, the equations of motion become (see, for example, [11])

$$
\begin{aligned}
& {\left[\partial_{\perp}^{2}+H_{\mathrm{B}} \partial_{\mathrm{b}}^{2}\right] H_{\mathrm{A}}\left(\boldsymbol{x}_{\perp}, \boldsymbol{z}_{\mathrm{b}}\right)=q_{\mathrm{A}} \delta(A) ;} \\
& {\left[\partial_{\perp}^{2}+H_{\mathrm{A}} \partial_{\mathrm{a}}^{2}\right] H_{\mathrm{B}}\left(\boldsymbol{x}_{\perp}, \boldsymbol{z}_{\mathrm{a}}\right)=q_{\mathrm{B}} \delta(B) ;} \\
& \left(\partial_{\mathrm{b}} H_{\mathrm{A}}\right)\left(\partial_{\mathrm{a}} H_{\mathrm{B}}\right)=0 .
\end{aligned}
$$


From the last equation, it is clear that one clump of branes, say the A-branes, is necessarily delocalized along the worldvolume of the other clump. For the prototype D0 $\| \mathrm{D} 4$ this is no restriction because there are no $\boldsymbol{z}_{\mathrm{b}}$. However, for an orthogonal

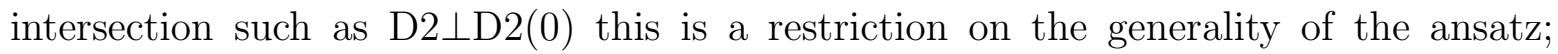
we will remark on the case of orthogonal intersections in the last section. Relabeling the $\mathrm{D} p+4$ brane the 'big' brane and the $\mathrm{D} p$ the 'wee' one, and $\boldsymbol{z}_{\mathrm{a}} \equiv \boldsymbol{z}$, our basic supergravity equations become

$$
\begin{aligned}
& {\left[\partial_{\perp}^{2}\right] H_{\mathrm{big}}\left(\boldsymbol{x}_{\perp}\right)=q_{\mathrm{big}} \delta(\mathrm{big}) ;} \\
& {\left[\partial_{\perp}^{2}+H_{\mathrm{big}}\left(\boldsymbol{x}_{\perp}\right) \partial_{z}^{2}\right] H_{\text {wee }}\left(\boldsymbol{x}_{\perp}, \boldsymbol{z}\right)=q_{\text {wee }} \delta(\text { wee }) .}
\end{aligned}
$$

The first equation is the usual one for the big branes. The second equation is more difficult to solve; here we will use the method of Surya and Marolf [2].

We can now pose the problem in which we are interested. Consider the setup of $N_{\text {big }}$ big branes with $N_{\text {wee }}$ wee branes separated from them in the transverse direction by $\boldsymbol{\Delta}$.

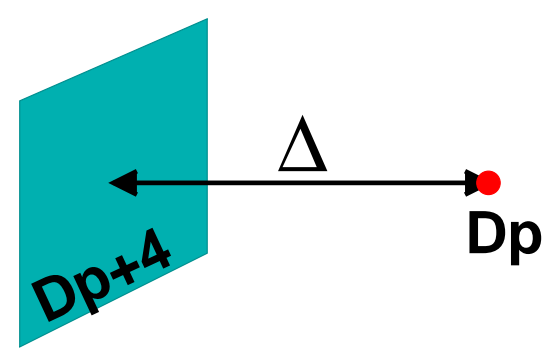

Figure 1: the setup for our problem of interest.

The $\mathrm{D} p+4$-branes are displaced from the $\mathrm{D} p$-branes by $\boldsymbol{\Delta}$.

What we wish to know is whether the wee brane can be localized in the worldvolume of the big brane as the separation is taken to zero, in the supergravity regime.

To motivate this, consider the simple and familiar example of the D-instanton in the background of the D3-brane. The supergravity solution is known explicitly only in the near-horizon region of the D3-brane, which is $\mathrm{AdS}_{5} \times \mathrm{S}^{5}$, [21] (see also [19, 20])

$$
\begin{aligned}
& \mathrm{d} S^{2}=H_{-1}\left(\operatorname{AdS}_{5} \times \mathrm{S}^{5}\right) ; \quad \exp (\Phi)=g_{\mathrm{s}} H_{-1} ; \quad \text { where } \\
& H_{-1}=1+\frac{3 N_{-1}}{16 \pi^{6} N_{3}^{2} g_{\mathrm{s}}^{2}} \frac{\rho^{4} \rho_{0}^{4}}{\left[\rho_{0}^{2}+\left|\boldsymbol{x}-\boldsymbol{x}_{\mathbf{o}}\right|^{2}\right]^{4}} .
\end{aligned}
$$

Here, the D-instanton position in Euclidean 10-space is $\left(\boldsymbol{x}_{\mathbf{o}}, \rho_{\mathbf{o}}\right)$, and the IR/UV relation relates $\rho$ to the usual radial coordinate $U=r / \ell_{\mathrm{s}}^{2}$ via

$$
\rho_{0}=\frac{\sqrt{4 \pi g_{\mathrm{s}} N}}{U_{0}}=\frac{\sqrt{4 \pi g_{\mathrm{s}} N \ell_{\mathrm{s}}^{4}}}{\Delta}=\frac{\mathcal{R}_{\mathrm{AdS}}^{2}}{\Delta} .
$$

The quantity $\rho_{0}$ is identified [21] as the scale size of the instanton in the gauge theory. Normally, the IR/UV relation is interpreted as saying that the instanton scale size $\rho_{0}$ goes to zero as the D-instanton goes to the boundary. In the context of our problem of interest, however, we are interested in the other end of things: as $\Delta \rightarrow 0, \rho_{0} \rightarrow \infty$. An alternative way to see that the D-instanton delocalizes without the AdS/CFT baggage 
is simply to stare at the supergravity harmonic function for the D-instanton: as the separation $\Delta \propto 1 / \rho_{0} \rightarrow 0, H_{-1}$ loses all information about the relatively transverse coordinates $\boldsymbol{x}$

$$
H_{-1} \quad \longrightarrow \quad 1
$$

This is a no-hair theorem as, if this $\boldsymbol{x}$ information had not been erased, we could have used the above process to build black D3-branes with D-instanton 'hair' in the form of an arbitrary distribution of D-instanton charge.

Note that in our analysis our solutions will always be taken to be such that they can be matched onto the full asymptotically flat solutions. This means that in any $p$-brane AdS/CFT correspondence we are doing gauge theory on $R^{p}$. As mentioned in [1], this condition rules out some known near-horizon supergravity solutions [22]; it would be interesting to learn what interpretation these solutions may have in another context. At this point we also mention that there are some other known solutions which are not of the type we want. Some are partially localized, but typically these are Büscher T-dual to larger-brane solutions. In addition, from the second equation of motion in (2.3) it is possible to see that we can trade some transverse localization for worldvolume localization. Since this does not maintain the integrity of the dimension of bigger brane in the solution, we will not consider these either.

Let us recap the solution for the harmonic function of the big $(\mathrm{D} p+4)$ branes. We have

$$
\partial_{\perp}^{2} H_{\mathrm{big}}=q_{\mathrm{big}} \delta\left(\boldsymbol{x}_{\perp}\right) \quad \Rightarrow \quad H_{\mathrm{big}}=1+\frac{c_{\mathrm{p}+4} g_{\mathrm{s}} N_{\mathrm{p}+4}}{\left|\boldsymbol{x}_{\perp}\right|^{3-p}}
$$

where $c_{\mathrm{p}}=(2 \pi)^{(5-p) / 2} \Gamma[(7-p) / 2]$. For the wee branes,

$$
\left[\partial_{\perp}^{2}+H_{\text {big }}\left(\boldsymbol{x}_{\perp}\right) \partial_{z}^{2}\right] H_{\text {wee }}\left(\boldsymbol{x}_{\perp}, \boldsymbol{z}\right)=q_{\text {wee }} \delta\left(\boldsymbol{x}_{\perp}-\boldsymbol{\Delta}\right) \delta(\boldsymbol{z}) .
$$

Surya and Marolf's method [2] for solving this equation of motion involves doing a Fourier transform in the four relatively transverse $\boldsymbol{z}$ coordinates, and it allows transverse separation $\boldsymbol{\Delta}$. The Fourier transform converts the $\boldsymbol{z}$ derivative into a wavevector, and the equation can then be solved Fourier mode by Fourier mode. It can also be shown that in order to track the behaviour of each Fourier mode at small separation $\boldsymbol{\Delta}$, keeping the spherically symmetric modes suffices. Then the equation reduces to a second order ODE in the radial variable $r=\left|\boldsymbol{x}_{\perp}\right|$; this can be solved explicitly and matched across the $\delta$-function. The resulting Fourier series/transform can be shown to converge absolutely, except of course at the location of the $\delta$-function. For details, the reader is referred to the original paper [1]].

The results obtained by this method give distinctly different behaviour depending on $p$ [1]:

$$
\begin{array}{cl}
-1 \leq p \leq 1: & \text { baldness } \\
p=2: & \text { hair } .
\end{array}
$$

(Recall that we are not going higher than $p=2$ because we take the big-brane, which has dimension $p+4$, to be asymptotically flat.) For a previous idea on expectations based on the dimensionality $p$ see [23]. 
From the supergravity perspective these hair/baldness results come about because of the different character of the differential equations with $1 / r$ versus stronger potentials $1 / r^{n>1}$. Note also that the result for $p=2$ meshes nicely with the existence of an explicit analytic solution found in [18], viz. D2-branes inside D6-branes, in the near-horizon limit of the D6-brane.

We can actually do better than deriving no-hair theorems for the low-dimensional cases; we can also work out the rates of delocalization of the wee-branes as they approach the big-branes. These may be computed by sitting out at a fixed transverse distance from the big-brane, which we choose to be the characteristic supergravity radius $R_{p+4}=\ell_{\mathrm{s}}\left(g_{\mathrm{s}} N_{\mathrm{big}}\right)^{1 /(3-p)}$, and watching the behaviour of the different Fourier modes of the fields of the wee-brane in the $\boldsymbol{\Delta} \rightarrow 0$ limit. The delocalization can thereby be characterized by a distance scale $\delta z$. From the supergravity equations we thusly find the scaling [1]

$$
\begin{array}{ll}
p=-1: & \frac{\delta z}{\ell_{\mathrm{s}}} \sim\left(g_{\mathrm{s}} N_{3}\right)^{1 / 2}\left[\frac{\ell_{\mathrm{s}}}{\Delta}\right] \\
p=0: & \frac{\delta z}{\ell_{\mathrm{s}}} \sim\left(g_{\mathrm{s}} N_{4}\right)^{1 / 2}\left[\frac{\ell_{\mathrm{s}}}{\Delta}\right]^{1 / 2} ; \\
p=1: & \frac{\delta z}{\ell_{\mathrm{s}}} \sim\left(g_{\mathrm{s}} N_{5}\right)^{1 / 2}\left\{\log \left[\frac{\left(g_{\mathrm{s}} N_{5}\right)^{1 / 2} \ell_{\mathrm{s}}}{\Delta}\right]\right\}^{1 / 2} .
\end{array}
$$

The result for the D-instanton agrees with the IR/UV relation, which is a consistency check. The other relations look suspiciously like low-dimensional quantum field theory relations, and we will confirm this later in a precise fashion. We will also see why the no-hair theorems depend so strongly on the dimension $p$.

\section{The AdS/CFT correspondence and the CMW theorem}

We would now like to define an AdS/CFT correspondence for this system of two types of branes by analogy with [3, 24; see also 225. Of course, the gauge couplings on the big and wee branes are built out of the same string theory parameters, $g_{\mathrm{YM}}^{2(p)}=(2 \pi)^{p-2} g_{\mathrm{s}} \ell_{\mathrm{s}}^{p-3}$. The decoupling limit à la Maldacena can be cast in dimensionless statements as follows: $\left(E \ell_{\mathrm{s}}\right) \rightarrow 0, E \sim U=r / \ell_{\mathrm{s}}^{2} \sim \Delta / \ell_{\mathrm{s}}^{2}$. Now, since the dimensionless gauge coupling is formed by factoring in $p-3$ powers of the typical gauge theory energy $E$ [24],

$$
g_{\mathrm{eff}}^{2}=(2 \pi)^{p-2} g_{\mathrm{s}}\left(E \ell_{\mathrm{s}}\right)^{p-3},
$$

the physics in the low-energy or decoupling limit will depend on which dimensionless coupling we keep fixed. Since we wish to keep the physics of the big-brane in the game, we keep the big-brane coupling fixed and so the wee-brane coupling will go to infinity in this limit, at 'fixed' energy E. Simply put, the big-brane physics is irrelevant in the infrared. Thus, the field theory that we will be interested in is $p+1$ dimensional.

We also need to pin down the other parameters. Since we want to be in the supergravity regime, we will keep $N_{\text {wee }}, N_{\text {big }} \gg 1$ but introduce no particular hierarchy 
between them. We also choose to keep the relative four-volume $V_{4}$ 'fixed', i.e. we will not scale it to zero as $\ell_{\mathrm{s}}^{4}$. We are most interested in the case where the big-brane is non-compact, i.e. $V_{4} \rightarrow \infty$. In the context of the $\mathrm{AdS}_{5} \times \mathrm{S}^{5}$ correspondence, this means having only a finite number of D-instantons. However, in our supergravity analysis we can also handle the case of finite-volume, which in the $\mathrm{AdS}_{5} \times \mathrm{S}^{5}$ case corresponds to a finite density of D-instantons per unit D3-worldvolume; see [26] for an analysis of this system.

\subsection{The supergravity side}

Taking the decoupling limit of our supergravity solution makes the big $(p+4)$-brane go near-horizon; we lose the 1 in its harmonic function $H_{\mathrm{big}}$,

$$
H_{\mathrm{big}} \longrightarrow \frac{1}{\ell_{\mathrm{s}}^{4}} \frac{c_{\mathrm{p}+4}}{(2 \pi)^{p+2}} \frac{N_{\mathrm{p}+4} g_{\mathrm{YM}}^{2(p+4)}}{U^{3-p}} .
$$

The more interesting issue is what happens to the wee-brane. Our solution retains dependence on the separation $\boldsymbol{\Delta}$. For the cases which delocalize, we can analyze the harmonic function for the wee-brane near the core of the big-brane. We find by careful attention to boundary conditions that we must not drop the 1 in the harmonic function for the delocalizing wee-brane [1]. See also [27] for similar conclusions on this issue. This phenomenon of not losing the 1 we saw very explicitly for the case of the D-instanton in the near-horizon region of the D3-brane, again a consistency check. (For the $p=0$ system, in a sense we are operating "half-way" between a probe calculation where $H_{0}=1$ and a DLCQ calculation where the 1 in the supergravity $H_{0}$ is dropped at the outset.)

In addition, upon checking the dilaton and metric curvatures, we find that the delocalizing wee-brane has a minor effect [1]; it does not change the region where the curvature becomes large, and the region where the dilaton becomes large occurs only extremely close to the wee-branes - the presence of the big-brane transversely shrinks the strong-dilaton region that occurs for $N_{\text {big }}=0$.

Note also that for the case of finite $V_{4}$ it turns out that, as $\boldsymbol{\Delta} \rightarrow 0$, we recover the smeared solution in which $H_{\text {wee }} \propto 1 / U^{3-p}$ just like $H_{\text {big }}$.

\subsection{The field theory side}

In the decoupling limit, the coupling between bulk and brane physics vanishes. In the $p+1$ dimensional field theory, decoupling occurs between the Higgs and Coulomb branches, even quantum mechanically 28]. For our situation where we are near-horizon for the big-brane, we are in the Higgs branch of the field theory. The field content may be recalled by remembering that the $\mathrm{D} p$ branes can be thought of as instantons in the $\mathrm{D} p+4$-brane; the $p+1$ dimensional field theory is the theory of the collective modes of these instantons. As reviewed in [29] (see also [30, [31]), the fact that the wee-brane

gauge coupling goes to infinity at fixed energy in our scaling limit means that some fields, which correspond to the $p-p$ strings which are transverse scalars, become auxiliary and 
may be integrated out. The resulting theory is a nonlinear sigma-model. (See also [32] for an analysis in a different regime, where different fields may be integrated out.)

The degrees of freedom of our Higgs branch theory are the instanton scale size and orientation, and center of mass position. In addition, the theory is very strongly coupled because we are working in the supergravity regime. Let us concentrate on the instanton scale size mode, which take as a proxy for how delocalized the wee-brane is. In low-dimensional quantum field theories, the Coleman-Mermin-Wagner theorem prevents superselection sectors in the quantum regime. This theorem then tells us that we have wild infrared fluctuations leading to complete delocalization for $p \leq 1$, i.e. baldness. For $p \geq 2$ superselection sectors are allowed quantum mechanically, so that we have hair for $p=2$. These results are in direct accord with the supergravity results, as we promised earlier.

In fact, we can go further and use scaling arguments to compute in the lowdimensional field theories estimates for the delocalization rates. As the separation $\Delta \rightarrow 0$, the IR fluctuations will become stronger and stronger, and eventually give complete delocalization. Let us take the IR cutoff to be

$$
\Lambda_{\mathrm{IR}}=\frac{\Delta}{\ell_{\mathrm{s}}^{2}} .
$$

In a sense we are reconstructing a field that was integrated out by putting back this IR cutoff; see also [29] for a more quantitative discussion on this point.

We now wish to calculate a rms value for the instanton scale size. We first treat the case of the D0-brane inside the D4-brane. For a single D0 instanton the moduli space metric is flat; we will assume that for large- $N_{0}$ the free-field approximation works as well, possibly modulo $1 / N$ corrections which we will not worry about. Also we assume that each instanton fluctuates independently but has approximately the same size - this latter assumption is motivated by the work of [33] on D-instantons in the original $\mathrm{AdS}_{5} / \mathrm{CFT}_{4}$ correspondence. Then, roughly speaking, since each instanton has $N_{4}$ directions in the gauge group in which to point, since the action has a normalization factor $g_{\mathrm{s}} \ell_{\mathrm{s}}$, and introducing $\Lambda_{\mathrm{IR}}$ by dimensional analysis gives

$$
\begin{aligned}
&\left\langle\rho^{2}\right\rangle \sim N_{4} g_{\mathrm{s}} \ell_{\mathrm{s}} \Lambda_{\mathrm{IR}}^{-1}, \quad \text { i.e. } \\
&\left.\frac{\sqrt{\left\langle\rho^{2}\right\rangle}}{\ell_{\mathrm{S}}}\right|_{\mathrm{D} 0} \sim\left(g_{\mathrm{s}} N_{4}\right)^{1 / 2}\left[\frac{\ell_{\mathrm{s}}}{\Delta}\right]^{1 / 2} .
\end{aligned}
$$

This agrees with the supergravity result, as we are taking the rms instanton scale size as a proxy for $\delta z$. It also agrees with a relation derived in 29]. (We remark here that this delocalization is not in contradiction with localization of center of mass modes for wee-branes without big-branes present which occurs in accordance with the existence of localized supergravity solutions for wee-branes by themselves. The difference in the current context is partly in a factor of order $1 / N_{\text {wee }} \ll 1$. So even though it is possible that the CM modes may be localized for the wee-brane instantons inside the big-branes, the fact that the scale sizes are totally delocalized is enough to conclude that we have agreement with the supergravity results.) 
For the $p=1$ case, by similar reasoning we get

$$
\left\langle\rho^{2}\right\rangle \sim N_{5} g_{\mathrm{s}} \ell_{\mathrm{s}}^{2} \log \left[\frac{\Lambda_{\mathrm{UV}}}{\Lambda_{\mathrm{IR}}}\right] .
$$

Taking

$$
\Lambda_{\mathrm{UV}} \sim \frac{1}{\ell_{\mathrm{s}}},
$$

yields, to the accuracy of our scaling analysis,

$$
\left.\frac{\sqrt{\left\langle\rho^{2}\right\rangle}}{\ell_{\mathrm{s}}}\right|_{\mathrm{D} 1} \sim\left(g_{\mathrm{s}} N_{5}\right)^{1 / 2}\left\{\log \left[\frac{\left(g_{\mathrm{s}} N_{5}\right)^{1 / 2} \ell_{\mathrm{s}}}{\Delta}\right]\right\}^{1 / 2},
$$

which again agrees with supergravity. In this case, renormalization effects (which are absent for $p<1$ ) complicate the comparison with [29].

Although the case of the D-instanton is rather degenerate, in that the field theory is zero-dimensional, the scaling analysis

$$
\left\langle\rho^{2}\right\rangle \sim N_{3} g_{\mathrm{s}} \Lambda_{\mathrm{IR}}^{-2},
$$

gives the scale-radius duality relation back again, up to constants:

$$
\left.\frac{\sqrt{\left\langle\rho^{2}\right\rangle}}{\ell_{\mathrm{s}}}\right|_{\mathrm{D}-1} \sim\left(g_{\mathrm{s}} N_{3}\right)^{1 / 2}\left[\frac{\ell_{\mathrm{s}}}{\Delta}\right] .
$$

This is a consistency check on our method.

We have seen that the supergravity solutions for the parallel intersections are capable of reproducing the field theory behaviour, which is of course very sensitive to the dimension $p$. Conversely, the field theory analysis is capable of detecting supergravity no-hair theorems.

\section{Orthogonal intersections}

So far we have discussed the results of [1] only for the prototypical cases $\mathrm{D} p \| \mathrm{D} p+4$. We now discuss briefly the case of orthogonal intersections, and for purposes of illustration we discuss the example of $\mathrm{D} 2 \perp \mathrm{D} 2(0)$.

The field theory analysis of the previous section suggests that we can draw hair/baldness conclusions for the near-horizon geometry of the orthogonal intersections as well, by T-duality. What matters is the dimensionality $p$ of the intersection theory. For example, for the D2 $\perp \mathrm{D} 2(0)$ system which has a 0-dimensional intersection, we expect baldness. More specifically, the mode that used to be the instanton scale size becomes, under T-duality, what might be termed the 'blow-up' mode. If we trade the two worldvolume coordinates in each clump of D2-branes (A and B) for a single complex coordinate $w$ so that we have $w_{\mathrm{A}}, w_{\mathrm{B}}$, then the 'blowup' mode $\rho$ appears in the combined worldvolume equation as $w_{\mathrm{A}} w_{\mathrm{B}}=\rho^{2}$.

Now, in the supergravity regime, we have delocalization, but this applies only to the near-horizon region of the geometry. Let us assume that there is no subtlety in matching 
this near-horizon part onto the asymptotically flat part, as the supergravity equations of motion suggest. Then, we can draw conclusions about asymptotically flat supergravity solutions as well. Far away from the intersection, we expect each clump of D2-branes to be localized, as they would be if the other clump were not present. Combined with smearing near the intersection, this gives an overall picture of a supergravity geometry with a 'fat neck'.

More generally, for the solutions with intersection dimensionality $p \geq 2$, we expect to be able to have a skinny neck, but if $p \leq 1$, we expect a fat neck.

The ansatz we made in setting up our supergravity analysis will not cover the case of fully localized orthogonal intersections, because it requires one clump of branes to be delocalized in the worldvolume of the other clump. Some progress has been made in generalizing the ansatz, see for example [15], but to our knowledge the only explicit solutions obtained so far are perturbative [34]. In that analysis, the ansatz [15] was used, along with source terms motivated by 'BIon' physics of [35, 36] and the solutions were obtained to second order. For $p \leq 1$ the second-order perturbations were divergent, even

after very careful attention to boundary conditions and singularities, while for $p \geq 2$ they were well-behaved. These results lend support to our field theory expectations. We await future progress in order to hear the final verdict, e.g. to find out 'how fat is fat'.

The search for various fully localized supergravity solutions is difficult. We would like to advocate the position that some of these solutions may not be found because there is a good field theory reason for them not to exist.

It would be interesting to see how far the results of our work can be generalized. One area of interest is nonextremal systems; work on this is in progress.

\section{Acknowledgments}

The author wishes to thank Don Marolf for collaboration on the work presented at the conference. In addition, we wish to thank Ofer Aharony, Micha Berkooz, Per Kraus, Joseph Polchinski, and Herman Verlinde for helpful discussions.

\section{References}

[1] D. Marolf and A.W. Peet 1999, to be published in Phys. Rev. D, e-print archive: hep-th/9903213.

[2] S. Surya and D. Marolf 1998, Phys. Rev.D 58, 124013 (e-print archive: hep-th/9805121).

[3] J.M. Maldacena 1998, Adv. Theor. Math. Phys. 2, 231 (e-print archive: hep-th/9711200).

[4] N.D. Mermin and H. Wagner 1966, Phys. Rev. Lett. 17, 1133.

[5] S. Coleman 1973, Commun. Math. Phys. 31, 259.

[6] R. Khuri 1993, Phys. Rev. D48, 2947.

[7] G. Papadopoulos and P.K. Townsend 1996, Phys. Lett. B B380, 273 (e-print archive: hepth/9603087). 
[8] A.A. Tseytlin 1996, Nucl.Phys. B475, 149 (e-print archive: hep-th/9604035).

[9] J.P. Gauntlett, D.A. Kastor, and J. Traschen 1996, Nucl. Phys. B478, 544 (e-print archive: hepth/9604179).

[10] A.A. Tseytlin 1997, Class. Quant. Grav. 14, 2085 (e-print archive: hep-th/9702163).

[11] H. Lu and C.N. Pope 1998, Int. J. Mod. Phys. A13, 4425 (e-print archive: hep-th/9710155).

[12] I. Ya. Aref'eva, M. G. Ivanov, O. A. Rytchkov, and I. V. Volovich 1998, Class. Quant. Grav. 15, 2923 (e-print archive: hep-th/9802163).

[13] J.P. Gauntlett 1997, Lectures given at APCTP Winter School "Dualities of Gauge and String Theories", Korea, February 1997, e-print archive: hep-th/9705011.

[14] H. Yang 1999, e-print archive: hep-th/9902128.

[15] A. Fayyazuddin and D. J. Smith 1999, J. High Energy Phys. 9904, 030 (e-print archive: hepth/9902210).

[16] A. Loewy 1999, e-print archive: hep-th/9903038.

[17] A. Hashimoto 1999, J. High. Energy Phys. 9901, 018 ( e-print archive: hep-th/9812159).

[18] N. Itzhaki, A. Tseytlin, and S. Yankielowicz 1998, Phys. Lett. B 432, 298 (e-print archive: hepth/9803103).

[19] C-S. Chu, P-M. Ho, and Y-Y Wu 1999, Nucl.Phys. B541, 179 (e-print archive: hep-th/9806103).

[20] I. Kogan and G. Luzon 1999, Nucl. Phys. B539, 121 (e-print archive: hep-th/9806197).

[21] M. Bianchi, M. Green, S. Kovacs, and G. Rossi 1998, J. High Energy Phys. 9808, 013 (e-print archive: hep-th/9807033).

[22] D. Youm 1999, e-print archive: hep-th/9902208.

[23] O. Pelc and R. Siebelink 1999, e-print archive: hep-th/9902045.

[24] N. Itzhaki, J. M. Maldacena, J. Sonnenschein, and S. Yankielowicz 1998, Phys. Rev. D58, 046004 (e-print archive: hep-th/9802042).

[25] H.J. Boonstra, B. Peeters and K. Skenderis 1998, Nucl. Phys.B533 127 (e-Print Archive: hepth/9803231).

[26] A. Tseytlin and H. Liu 1999, Nucl.Phys.B553, 231 (e-print archive: hep-th/9903091).

[27] E. Martinec and V. Sahakian 1999, e-print archive: hep-th/9906137.

[28] E. Witten 1996, in Proceedings of Strings '95, ed. by I. Bars et al, World Scientific, Singapore, 1996 (e-print archive: hep-th/9507121); and 1997, J. High Energy Phys. 9707, 003 (e-print archive: hep-th/9707093).

[29] H. Verlinde and M. Berkooz 1999, e-print archive: hep-th/9907100.

[30] O. Aharony, M. Berkooz, S. Kachru, N. Seiberg, and E. Silverstein 1998, Adv. Theor. Math. Phys. 1148 (e-print archive: hep-th/9707079).

[31] N. Seiberg and E. Witten 1999, J. High Energy Phys. 9904, 017 (e-print archive: hep-th/9903224).

[32] O. Aharony and M. Berkooz 1999, e-print archive: hep-th/9909101.

[33] N. Dorey, T.J. Hollowood, V.V. Khoze, M.P. Mattis, S. Vandoren 1999, Nucl. Phys.B 552, 88 (e-Print Archive: hep-th/9901128).

[34] A. Gomberoff, D. Kastor, D. Marolf and J. Traschen 1999, e-print archive: hep-th/9905094.

[35] C.G. Callan Jr. and J.M. Maldacena 1998, Nucl. Phys.B513, 198 (e-Print Archive: hepth/9708147).

[36] G.W. Gibbons 1998, Nucl. Phys.B514, 603 (e-Print Archive: hep-th/9709027). 